\title{
Discrimination of EEG-Based Motor Imagery Tasks by Means of a Simple Phase Information Method
}

\author{
Ana Loboda \\ Faculty of Medical Bioengineering \\ "Grigore T. Popa" University of Medicine and Pharmacy \\ Iasi, Romania \\ Gabriela Rotariu \\ Faculty of Medical Bioengineering \\ "Grigore T. Popa" University of Medicine and Pharmacy \\ Iasi, Romania
}

\author{
Alexandra Margineanu \\ Faculty of Medical Bioengineering \\ "Grigore T. Popa" University of Medicine and Pharmacy \\ Iasi, Romania
}

Anca Mihaela Lazar

Faculty of Medical Bioengineering

"Grigore T. Popa" University of Medicine and Pharmacy Iasi, Romania

\begin{abstract}
We propose an off-line analysis method in order to discriminate between motor imagery tasks manipulated in a brain computer interface system. A measure of large-scale synchronization based on phase locking value is established. The results indicate that it can take advantage of the phase synchrony between scalp-recorded EEG activity in the supplementary motor area and in sezorimotor area, computing the differences between the active and the relaxation states. Phase locking value features are more discriminative in $\beta$ rhythm than in $\mu$ rhythm. The proposed method is simple, computationally efficient and proves good results on EEG Motor Movement/Imagery Dataset available from PhysioNet research resource for physiologic signals.
\end{abstract}

Keywords-brain computer interface; motor imagery task; electroencephalogram; phase locking value

\section{INTRODUCTION}

Brain computer interface (BCI) is a computerized system that acquires brain signals generated during various mental tasks, extracts and classifies suitable features, translates into appropriate control signals and sends them to an external device. BCI represents a real help for people with motor disabilities.

Various methods for monitoring brain activity (electroencephalogram - EEG, magnetoencephalogram - MEG, positron emission tomography - PET, functional magnetic resonance imaging - fMRI, single photon emission computed tomography - SPECT) can represent, by itself, the base of a brain computer interface. Taking into account the low volume and the low cost of the equipment, the simple preparation for registration, the possibility of portability, the real-time analysis, EEG is a method with certain advantages against all the others. So, it is frequently used for implementation of the BCIs.

The EEG is a non-invasive method for recording the electrical activity of the brain, using surface electrodes placed on the scalp.

According to the type of mental activities, the BCI classification is as follows:
- BCI based on evoked responses such as P300 potential, visual evoked potential;

- Operant conditioning BCI such as BCI that uses changes in cerebral rhythms, BCI using slow cortical potentials, BCI using other areas of the cortex specialized for different mental activities [1].

This research is focused on the paradigm of using as BCI discriminating tasks changes in cerebral rhythms, when a subject move or think of movement of left/right hand.

When a mental activity is produced, such as preparation, execution or imagination of hand movement, changes in the corresponding signal power of $\mu(8-12 \mathrm{~Hz})$ and $\beta$ rhythms (12$30 \mathrm{~Hz}$ ) occur. These changes are known as event related desynchronization (ERD) and event related synchronization (ERS) of these rhythms. It was found that imagining, preparation or planning of movement develops the same kind of brain activity, ERD or ERS, in the same brain regions. When a subject intends to move or imagines to move the right or left hand, there is a short period of $\mu$ or $\beta$ rhythm ERD in the opposite hemisphere of the brain. After the action period, a $\mu$ or $\beta$ ERS occurs also in the opposite hemisphere of the part of the body imagined moving [2].

The main signal processing methods used in a motor imagery task paradigm are: power spectral density, parametric analysis based on autoregressive method, correlation, short Fourier transform, continuous and discrete wavelet transform [3]. Although the methods that exploit the connectivity between different regions of the brain are not so frequently used, there are some interesting papers dealing with the study of rhythms associated to motor imagery in BCI by means of phase information of the EEG signal [4], [5], [6].

We focus our attention on such a method linked with the synchronization between EEG signals from different area of the brain. Our aims are:

- to establish an algorithm suitable to decide with a high rate of success if the proposed EEG phase feature (recorded by few channels placed on the both side of 
the scalp) occurs when the subject has moved his left hand or when he/she moved his/her right hand;

- to see what is the rhythm that produce the better feature selection in order to discriminate between the above mentioned movement tasks.

We perform an off-line analysis in order to measure the large-scale synchronization by means of the phase locking value (PLV). Contrary to power, we expect synchrony in $\mu$ or $\beta$ rhythms to be greater in the contralateral hemisphere (with respect to the target direction).

\section{METHOD}

There are several methods of measuring the synchronization between two signals $\mathrm{x}(\mathrm{t})$ and $\mathrm{y}(\mathrm{t})$. Among these methods there are worth to be mentioned phase crosscoherence analysis, mutual information, Shannon entropy, synchronization likelihood and phase locking value.

PLV is a measure of synchronization in the time domain and it is applied for analysis of EEG signals recorded during performing a motor imagery task.

PLV characterizes the stability of the phase difference between instantaneous phases $\varphi_{\mathrm{x}}(\mathrm{t})$ and $\varphi_{\mathrm{y}}(\mathrm{t})$ of signals $\mathrm{x}(\mathrm{t})$, respectively $\mathrm{y}(\mathrm{t})$ using the formula [4]:

$$
P L V=\left|\left\langle e^{j \Delta \varphi(t)}\right\rangle\right|
$$

where $\Delta \varphi(t)=\varphi_{y}(t)-\varphi_{x}(t)$ and $\langle$.$\rangle is average operator.$ Usually, the averaging is done on trials, but when there is only one trial or little trials, it is performed over time (number of samples for discrete signals). In this case, PLV has the following expression:

$$
P L V=\frac{1}{N}\left|\sum_{t=1}^{N} \exp \left[j\left(\varphi_{\mathrm{y}}(\mathrm{t})-\varphi_{\mathrm{x}}(\mathrm{t})\right)\right]\right|
$$

where $\mathrm{N}$ is the number of EEG samples of the trial.

When the phase difference is constant, PLV is equal to 1 . If the phase difference is distributed randomly in the interval $[0$, $2 \pi$ ], the phase difference follows a normal distribution, so PLV is equal to 0 .

In order to calculate the PLV, it is necessary to know the instantaneous phases $\varphi_{\mathrm{x}}(\mathrm{t})$ and $\varphi_{\mathrm{y}}(\mathrm{t})$. Instantaneous phases can be obtained using wavelet transform or computing analytic signal using Hilbert transform. It is proved that there are not important differences between methods and may be considered equivalent for study of EEG signals [7]. We have chosen to use the Hilbert transform.

The Hilbert transform was defined starting from causal signals, that there is a relationship between the real and imaginary parts of Fourier transform of the signals. The Hilbert transform of a signal $s(t)$ is given by the equation [4]:

$$
\tilde{s}(t)=\frac{1}{\pi} p \cdot v \cdot \int_{-\infty}^{+\infty} \frac{s(\tau)}{t-\tau} d \tau,
$$

where p.v. is the Cauchy principal value. The analytical signal is expressed as:

$$
S(t)=s(t)+j \tilde{s}(t)
$$

Instantaneous phase is calculated using the formula:

$$
\varphi(t)=\arctan \left(\frac{\tilde{s}(t)}{s(t)}\right) .
$$

It is known that there are two kind of synchrony in the brain: local-scale and large-range synchrony [8]. When dealing with adjacent channels in the same sensorimotor region of the brain it is said that local-scale synchrony may exist and when channels from widely regions are involved in computing synchrony it is said that large-scale synchrony may occur. As there are results proving best classification rates when using large-range synchronization [9], in this study we focus our attention only on this instance.

\section{RESULTS}

We used EEG Motor Movement/Imagery Dataset recorded using BCI2000 platform [10] available through Physionet [11]. We downloaded BCI2000 from www.bci2000.org. The database contains EEG recordings from 109 persons who performed various motor/imagery tasks. EEG recording was performed using 64 electrodes placed on the scalp according to the 10-20 extended international system (Fig. 1).

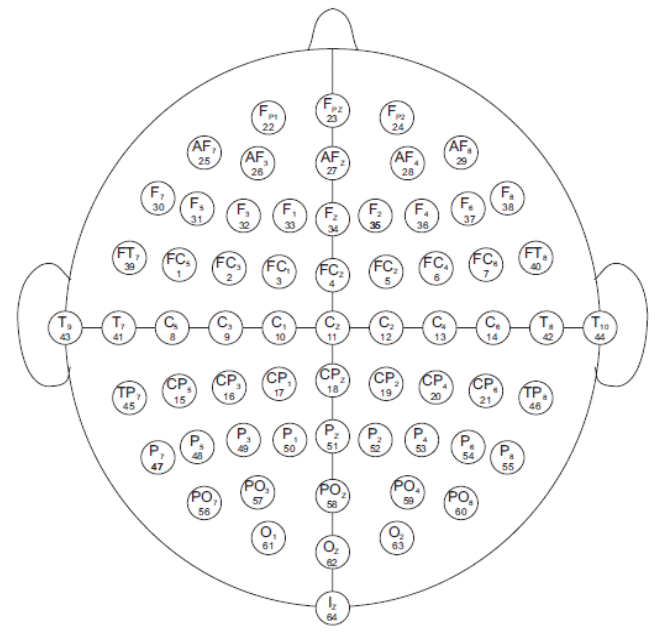

Fig. 1. Extended 10-20 international system of EEG electrodes placements

Every subject performed 14 experimental tasks: 2 runs of 1 minute for the relaxation state (one with closed eyes and one with eye open) and 3 runs of 2 minutes for each of the following tasks:

- Opening and closing the left/right fist when a target appears on the left/right side of the screen followed by relaxation.

- Imagining opening and closing the left/right fist when a target appears on the left/right side of the screen followed by relaxation.

- Opening and closing of both fists (if the target appears in the top of the screen) or of both feet (if the target appears on the lower side of the screen).

- Imagining opening and closing of both fists (if the target appears in the top of the screen) or of both feet (if the target appears on the lower side of the screen). 
We used only the appropriate sets of moving and imagining movement of opening and closing the left/right fist.

The EEG recorded signals of movement and imagining of movement were grouped into three data sets: for moving denoted by $3,7,11$ runs and for the imaginary denoted by 4, 8 , 12 runs. EEG signals were sampled at a frequency of $160 \mathrm{~Hz}$. We downloaded the EEG signals as mat files.

For each recorder there is a file with annotations of the moments when target appear in the left or the right side of the screen (indicating movement/imagining movement of right fist, left fist) and the periods of relaxation that occurs after each motor activity. Movement/imagining movement and relaxation are coded as follows: T0 relaxation period, T1 real movement/imaginary left fist, T2 real movement/imaginary right fist.

In what follows, we present the algorithm that is implemented both for real movement and for image movement of the fist. In each of these two circumstances, the study is focused on $8-12 \mathrm{~Hz}$ band of $\mu$ rhythm, then on $12-30 \mathrm{~Hz}$ of $\beta$ rhythm. At the end, a comparison between results is accomplished.

In the pre-processing stage, in each of the three runs of one state, the EEG signals segmentation simultaneous to the left/right motor task or relaxation periods is performed. We used the annotations to split each run into data segments which contained the portions of the experimental run consisting of $\mathrm{T} 1, \mathrm{~T} 2$ and T0 segments. Each segment is of $2 \mathrm{~s}$ interval, beginning with the $0.5 \mathrm{~s}$ after the moment when a T1/T2 or a T0 (after T1/T2) appears in the annotation file. At this step of the script, four 3 dimension arrays (number of trials by number of samples by number of channels) are returned: one for left movement, one for right movement and two arrays for relaxation (one following the left movement and one following the right movement period). We get 7 or 8 trials for each movement task and 7 or 8 trails for the relaxation periods. There are 320 samples and 64 channels. No artifact rejection method is performed. Then, a detrending method is applied.

As one of our goal is to emphasis the effect of the frequency band upon the discrimination of the left/right motor tasks, a band-pass filter is applied in each trial. In order to avoid phase distortion, a liner phase FIR filter is used.

After the pre-processing, the representative features are extracted. The Hilbert transform is performed for all the channels and the PLV is computed for all the possible pairs of EEG channels (in all states).

From all the pairs of electrodes, we employ three electrodes from the supplementary motor area, $\mathrm{FCz}, \mathrm{Cz}$ and $\mathrm{CPz}$, and other six ones from the sensorimotor areas, FC3, C3, CP3 (from left hemisphere), FC4, C4 and CP4 (from right hemisphere). Therefore, we extract nine PLVs for each hemisphere, that is FCz-FC3, FCz-C3, FCz-CP3, Cz-FC3, Cz$\mathrm{C} 3, \mathrm{Cz}-\mathrm{CP} 3, \mathrm{CPz}-\mathrm{FC} 3, \mathrm{CPz}-\mathrm{C} 3$ and $\mathrm{CPz}-\mathrm{CP} 3$ for left and $\mathrm{FCz}-$ FC4, FCz-C4, FCz-CP4, Cz-FC4, Cz-C4, Cz-CP4, CPz-FC4, $\mathrm{CPz}-\mathrm{C} 4$ and $\mathrm{CPz}-\mathrm{CP} 4$ for right hemisphere (as it is illustrated in Fig. 2).
For all these pairs, we compute the difference of PLVs between the active and relaxation periods as:

$$
P L V_{\text {diff }}=P L V_{m t}-P L V_{\text {rest }}
$$

where $P L V_{m t}$ is the average PLV over all the trials in the motor task and PLV rest is the average PLV over all the trials in the relaxation state.

In order to discriminate between left or right motor activity, we compare between PLV $V_{\text {diff }}$ of the corresponding pairs of electrodes from the left and right side and, according to the result of the comparison a vector of nine elements is built (one element for each comparison pairs). Each element of this vector may be 1 or 0 and is obtained in the following manner: if $P L V_{\text {diff left }}<P L V_{\text {diff right }}$ a value equal to 1 is put and, in the opposite case, a value equal to 0 . Hereafter, a majority vote is applied for classification into the two groups of the moving/imaging left fist and the moving/imaging right fist. As it is expected the synchrony is greater in the contralateral hemisphere, the decision is as follows: if the number of elements equal to 1 in the vector is greater or equal to 5 (a half of vector length plus 1) it means that the subject moved/imagined left fist and, on the contrary, the subject moved/imagined right fist.

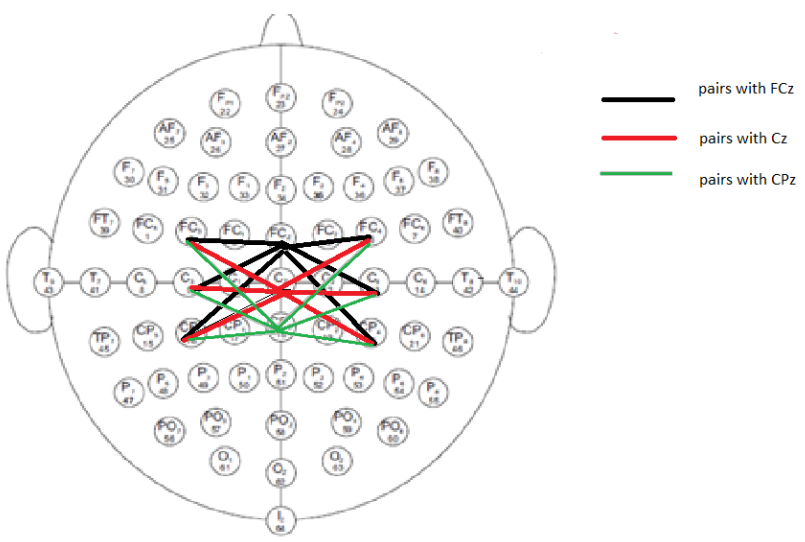

Fig. 2. The electrode pairs selection in the case of the large-range synchronization

In what follows, we denote by $100 \%$ success rate the case when, in the same run $(3,7$ or 11 for movement and 4,8 or 12 for imagery), according to our criterion, the decision that the subject moved/imagined the left/right fist corresponds for the EEG pattern extracted for the periods the target appears on the left, respectively on the right side of the screen. For example a $100 \%$ success rate is if in run 3, after the comparison of $P L V_{\text {diff left }}$ and $P L V_{\text {diff right }}$, for the movement of the target to the left side of the screen we get 6 values of 1 and for the right movement of the target we get 4 values equal to 0 . Then we conclude that the left fist was moved, respectively the right fist was moved.

We worked with the mentioned database of 109 volunteers, but only for 103 of them the mentioned algorithm was performed because for the other 6 subjects there are too short or damaged records. These invalid subjects are: S043, S088, S089, S092, S100 and S104. 
First of all, we focus on the implementation when the EEG signal is $8-12 \mathrm{~Hz}$ band-pass filtered.

The number of subjects with $100 \%$ success rate versus the number of runs is represented by bars in Fig. 3. The chart is for the two types of experiments, real and imagery movement of fist. In both circumstances, there are some subjects that have $100 \%$ success rate for all three runs (3, 7 and 11 for movement and 4, 8 and 12 for imagery), other subjects for two runs (any combinations of two runs between 3, 7 or 11 for movement or between 4,8 and 12 for imagery) and some subjects only for one run (any of 3, 7 or 11 for movement or any of 4, 8 or 12 for imagery). There are persons for whom, in the same run, the correct decision is only for the left or for the right motor task, but not simultaneously for left and right. There a cases when, in the same run, there is $100 \%$ success rate neither for the left nor for the right. For both of the last two situations, in the figure, there is mentioned by "none" on the abscise.

As we can notice from Fig. 3, there are not significant differences between the real movement and the imagery task. Besides, the number of $100 \%$ success rate for all the three runs is low, only 11/9 subjects from e total of 103 being able to correct discriminate in all runs for real movement, imagery movement, respectively. For the most of the subjects, the best result is only for two runs and one run. The worst situation, meaning that for none of the runs could be obtained $100 \%$ success rate, is for 23/21 subjects (real movement/imagery movement).

The 12- $30 \mathrm{~Hz}$ in another band at which we suspect phase locking to occur. So, we perform the same steps as in the previous case and chart from Fig. 4 shows the number of subjects with $100 \%$ success rate versus the number of runs.

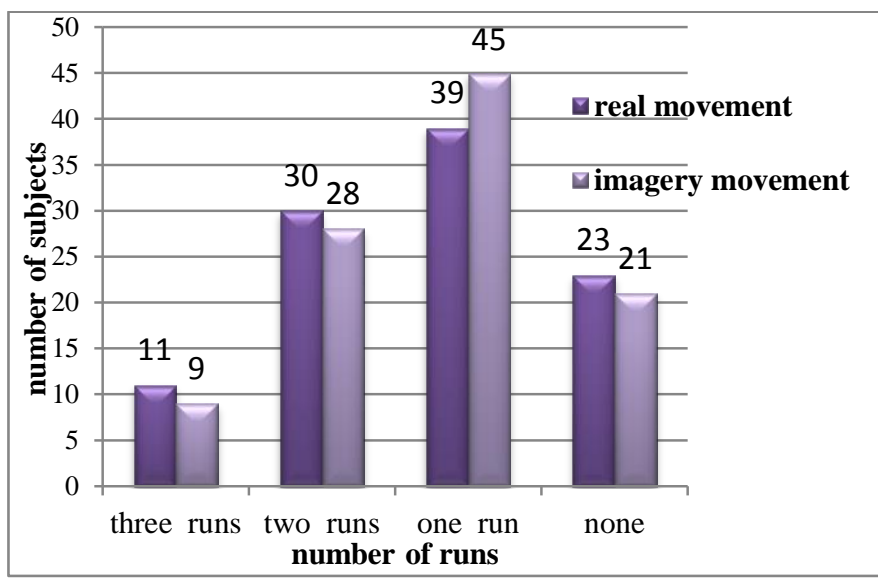

Fig. 3. Number of subjects with $100 \%$ success rate versus number of runs (EEG signal is 8-12 $\mathrm{Hz}$ band-pass filtered)

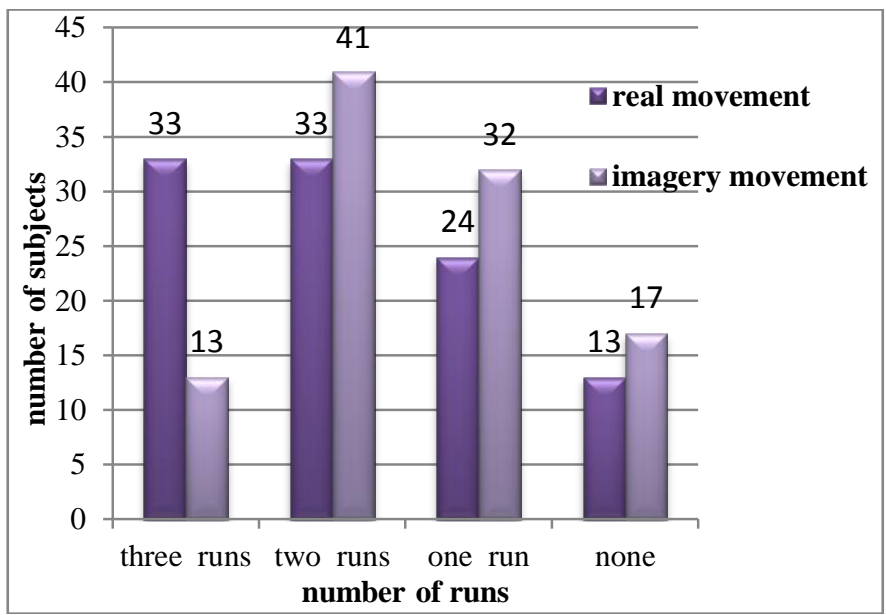

Fig. 4. Number of subjects with $100 \%$ success rate versus number of runs (EEG signal is $12-30 \mathrm{~Hz}$ band-pass filtered)

Comparing to Fig. 3, it is obvious that the greatest difference occurs for "three runs" in the case of real movement task, when the number of subjects who attend $100 \%$ success rate is three times higher ( 33 versus 11 subjects). A possible reason is that the phase locking for $\beta$ rhythm is more frequent than for $\mu$ rhythm. Concerning the imagery movement task, there is no significant difference between the subjects' number who attend $100 \%$ success rate for three runs in the case of the two different filter bands. We also observe that in the case of $12-30 \mathrm{~Hz}$ the number of subjects for two runs is greater than the number for one run. The number of subjects who attend $100 \%$ success rate for none of the runs is quite the same. It is possible that some of the volunteers are not able to perform the task or in the handled EEG patterns there are no discrepancies between relaxation and motor task.

It would be interesting if we compute the percentage of the left/right correct discrimination task reported to all the runs for all the subjects in the case of real movement and in imagery task. In Table 1 is depicted these results.

TABLE I. THE RATE OF CORRECT DisCRIMINATION TASK FOR ALL THE SUBJECTS

\begin{tabular}{|c|c|c|c|c|c|c|}
\hline \multirow[b]{3}{*}{$\frac{\mathrm{E}}{\mathrm{E}}$} & \multicolumn{6}{|c|}{ Side of movement/imagery } \\
\hline & \multicolumn{2}{|c|}{ Left } & \multicolumn{2}{|c|}{ Right } & \multicolumn{2}{|c|}{ Global } \\
\hline & 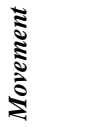 & 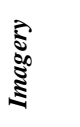 & 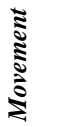 & 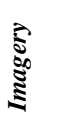 & 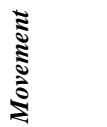 & 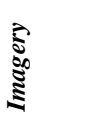 \\
\hline$\mu$ & $64.4 \%$ & $60.5 \%$ & $63.1 \%$ & $70.6 \%$ & $63.75 \%$ & $65.55 \%$ \\
\hline $\boldsymbol{\beta}$ & $75.4 \%$ & $68 \%$ & $82.5 \%$ & $75.1 \%$ & $78.95 \%$ & $71.55 \%$ \\
\hline
\end{tabular}


In the case we filtered on $8-12 \mathrm{~Hz}$ (corresponding to $\mu$ rhythm), when the subjects moved the fist, the results for the two kind of tasks, left or right movement, are quite the same, taking fair values, about $64 \%$. When the subjects imagined the movement, only for the right, the value is higher, about $70 \%$. Better results are when the filter was on $12-30 \mathrm{~Hz}$ (corresponding to $\beta$ rhythm), attaining $82.5 \%$ when right fist was moved.

When we compute the rate of classification in a global manner, that is considering all the matches between the position of the target (movement/imagery of the fist in that direction) and the decision of our classification method by the majority vote, we get better results for $\beta$ rhythm, than for $\mu$ rhythm both for movement $(78.95 \%$ versus $63.73 \%)$ and for imagery tasks $(71.55 \%$ versus $65.55 \%)$.

In order to compare our method with the others, it is correct to use the same database. To our concern, the already reported researches used only the amplitude change in EEG signals for feature selection. So, in [12], despite different investigated methods (per patient/per group, normalized by frequency band/not normalized by frequency band, independent component analysis ICA/non-ICA), the reported classification accuracies do not overpass $70 \%$, laying in $53.67 \%-69 \%$ interval. Taking into account these results and the fact that there were performed more difficult methods, our approach seems to be with certain advantages.

\section{CONCLUSIONS}

In the framework of BCI motor imagery paradigm, because phase is supposed to include most important information about the neural activity, in order to discriminate between left and right motor task, we have proposed a phase locking value based method.

The results suggest that this method can exploit, on one hand, the phase synchrony between scalp-recorded EEG activity in the supplementary motor area and in sezorimotor area and, on the other hand, the differences between the active and the relaxation states.

PLV features were more discriminative when computed from $12-30 \mathrm{~Hz}$ filtered EEG signals.

The algorithm is very simple and computationally efficient using only some suitable EEG channels.

Considering the fact that no trials are excluded due to artifacts, it is a promising method for further developments of BCI systems.

\section{DISCUSSION AND FUTURE WORK}

In this paper we considered the average over all the trails for the PLVs for computing PLV diff and, even if is time consuming, we have to test the method on each trial for every of the 103 subjects.

In order to be a valuable tool for BCI, we have to develop an appropriate method to discriminate in real time between the left and the right imagery movement.

As we used a large database, it was possible to report relevant results. There is a drawback using this database because we do not know details concerning the subjects or the experiment (e.g. the timing between runs) etc. So, we have to test the proposed method on our own EEG recordings in order to be able to explain results and establish connections to the variants that could influence them.

\section{REFERENCES}

[1] A.M., Lazăr, L. Davlea, R. Ursulean, A. Maiorescu, B. Teodorescu, Interfața creier-calculator- Paradigme posibile, CERMI, 2009 (in Romanian)

[2] G. Pfurtscheller, C. Neuper, "Motor Imagery and direct brain-computer communication", Proceedings IEEE, Vol. 89, No. 7, 1123-1134, 2001.

[3] A. Bashashati, M. Fatourechi, K. R. Ward and E. G., Birch "A survey of signal processing algorithms in brain-computer interfaces based on electrical brain signals", J. Neural Eng., 4, pp. 32-57, 2007.

[4] E. Gysels and P. Celka, "Phase synchronization for the recognition of mental tasks in a brain-computer interface", IEEE Transactions on neural systems and rehabilitation engineering, Vol. 12, No. 4, December 2004

[5] Le Song, E. Gordon, E. Gysels, "Phase synchrony rate for the recognition of motor imagery in brain-computer interface", Advances in Neural Information Processing Systems, vol. 18, pp. 1265-1272, 2006.

[6] D. Krusienski, D. McFarland, J. Wolpaw, "Value of amplitude, phase, and coherence features for a sensorimotor rhythm-based brain-computer interface", Brain Res Bull., vol. 87(1), pp. 130-134, Jan 4, 2012.

[7] M. Le Van Quyen et al., "Comparison of Hilbert transform and wavelet methods for the analysis of neuronal synchrony", Journal of Neuroscience Methods, 111, pp. 83-98, 2001.

[8] J. P. Lachaux, E. Rodriguez, J. Martinerie and F. J. Varela, "Measuring phase synchrony in brain signals", Hum. Brain Mapp., vol. 8, pp. 194208, 1999.

[9] Yijun Wang, Bo Hong, Xiaorong Gao, and Shangkai Gao "Phase synchrony measurement in motor cortex for classifying single-trial EEG during motor imagery", Proceedings of the 28th IEEE EMBS Annual International Conference New York City, USA, Aug 30-Sept 3, 2006.

[10] G. Schalk, D.J. McFarland, T. Hinterberger, N. Birbaumer, J.R. Wolpaw, "BCI2000: A general-purpose brain-computer interface (BCI) system.", IEEE Transactions on Biomedical Engineering 51(6), pp. 1034-1043, 2004.

[11] AL Goldberger et al., "PhysioBank, PhysioToolkit, and PhysioNet: Components of a New Research Resource for Complex Physiologic Signals." Circulation 101(23):e215-e220 [Circulation Electronic Pages; http://circ.ahajournals.org/cgi/content/full/101/23/e215]; June 13, 2000.

[12] J. Sleight, P. Pillai, and S. Mohan, "Classification of executed and imagined motor movement EEG signals," Ann Arbor: University of Michigan, pp. 1-10, 2009, Retrieved from http://www.scribd.com/doc/82045737/ICA. 